\title{
Hipertrofia ventricular izquierda con patrón de strain característico
}

\author{
Julián Vega ${ }^{1}$, Samuel Córdova ${ }^{1}$, Luigi Gabrielli ${ }^{1}{ }^{2},{\text { Paul } M c N a b^{1}}^{1}$, Rodrigo Saavedra ${ }^{1}$. \\ 1. División de Enfermedades Cardiovasculares. Escuela de Medicina. Pontificia Universidad Católica de Chile. \\ 2. Advanced Center for Chronic Diseases (ACCDiS), Escuela de Medicina, Pontificia Universidad Católica de Chile. \\ Sin apoyo financiero
}

\section{Left ventricular hypertrophy with a characteristic strain pattern}

A 68 year-old male, with no past medical history, presented progressive shortness of breath on exertion and leg edema during the previous year. Transthoracic echocardiography depicted; severe left ventricular hypertrophy with mild systolic dysfunction (LVEF 50\% biplane Simpson's method), severe diastolic dysfunction and moderate left atrial enlargement. Left ventricular myocardial deformation imaging by two-dimensional speckle-tracking was paramount for elucidating the differential diagnosis. Key words: Left Ventricular Hypertrophy, speckle tracking echocardiography. 
Hombre de 68 años, sin comorbilidades conocidas. Relata historia de 1 año de deterioro progresivo de la capacidad funcional hasta clase III NYHA, asociado a edema de extremidades inferiores. Se realiza una ecocardiografía transtorácica (EPIQ 7, Philips, Andover, MA, USA) describiendo; ventrículo izquierdo con severa hipertrofia concéntrica, disfunción sistólica global leve (FEVI 50\% por Simpson biplano), disfunción diastólica tipo III (E/A 3.0, E/e' medial 42, E/e' lateral 31), dilatación auricular izquierda moderada (volumen biplano indexado $45 \mathrm{ml} / \mathrm{m} 2$ ), insuficiencia mitral y tricuspídea leves, hipertensión pulmonar (presión sistólica de arteria pulmonar $42 \mathrm{mmHg}$ ) y ventrículo derecho de tamaño y función normal (Tabla 1). Debido a la sospecha de una miocardiopatía restrictiva se realiza estudio de la deformación miocárdica mediante el análisis del strain longitudinal por speckle tracking (bidimensional) del ventrículo izquierdo (VI). Para este fin se obtuvieron vista apicales de 4 cámaras, 3 cámaras y 2 cámaras que se procesaron mediante el software de cuantificación del movimiento cardíaco (CMQ Philips). Se objetivó una disminución del strain longitudinal global (S.L.G.) del VI: -12.3\% . Para una mejor interpretación de la deformación, se graficó el valor de strain longitudinal máximo de cada segmento miocárdico del VI en un mapa polar compuesto por 17 segmentos ( 6 basales, 6 medios, 4 apicales y el ápex verdadero), el cual se muestra en la Figura 1. Dicho mapa polar permitió observar un patrón de strain característico, contribuyendo a orientar la etiología de la hipertrofia ventricular y que se presenta como desafío diagnóstico al lector.

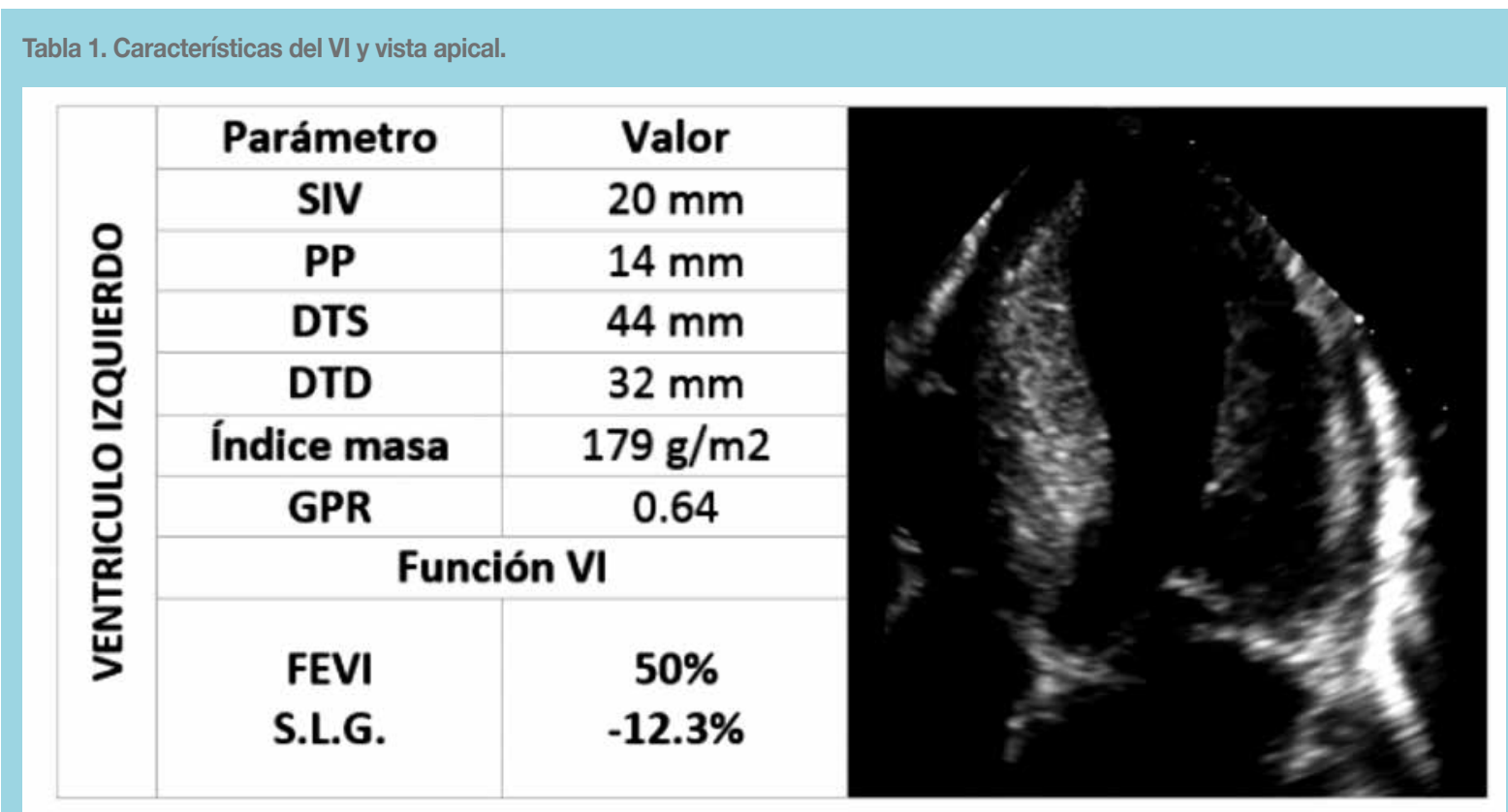

SIV: grosor del septum interventricular VI. PP: grosor de la pared posterior VI. Diámetro tele diastólico del VI. Diámetro tele sistólico del VI. GPR: Grosor relativo de la pared. FEVI: Fracción de eyección del ventrículo izquierdo por Simpson biplano; S.L.G.: strain longitudinal global 
www.ecocardio.cl

FC (Prom.) $=78 \mathrm{Ipm}$

VTD (Bi-plane) $=106.8$ ANTEROSEPTAL

VTS (Bi-plane) $=52.4 \mathrm{mi}$

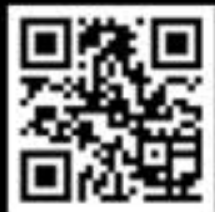

FE (Bi-plane) $=50.9$ ?

DesvEst tofro. $=51.7 \mathrm{~ms}$
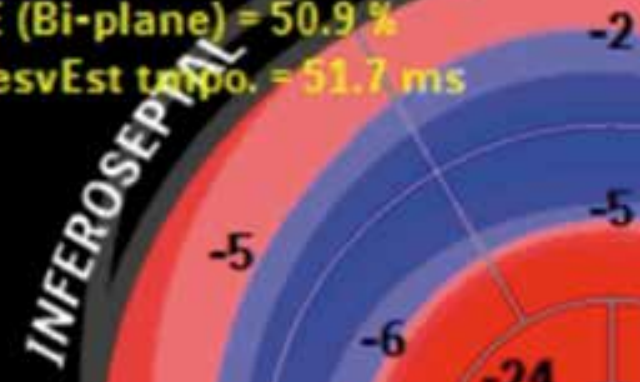

$-5$ $-2$
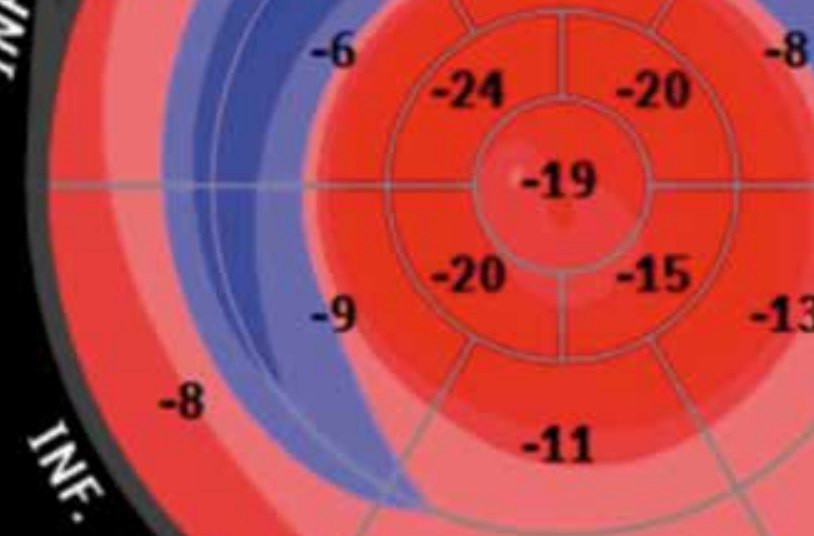

$-6$
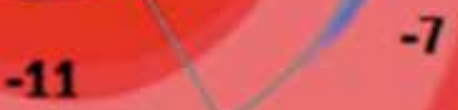

$-8$

A3C Deform. long. $=-1 / 1$ f8

A4C Deform. Iong. $=-13.2$ S ROLATERAL

A2C Deform. long. $=-12.7 \%$

Global Deform. long. $=-12.3 \%$ 


\section{Evolución:}

El paciente fue tratado con infusión de Milrinona y terapia depletiva con buena respuesta clínica. Dentro del estudio se realizó electroforesis de proteínas en sangre y orina y biopsias con estudio inmunohistoquímico de piel y de médula ósea, exámenes que corroboraron la sospecha ecocardiográfica, confirmándose el diagnóstico de Amiloidosis sistémica secundaria a mieloma múltiple (IgA Lambda).

\section{Discusión:}

La deformación miocárdica mediante el strain longitudinal por speckle tracking es una herramienta que aporta información adicional sobre la función ventricular izquierda, refleja la capacidad de acortamiento de la fibra miocárdica y tiene mayor sensibilidad que la fracción de eyección, permitiendo detectar alteraciones de la contractilidad de forma precoz y subclínica ${ }^{1}$. Por otra parte, la evaluación de la función sistólica global mediante el método de Simpson biplano tiende a sobreestimar la función sistólica en casos de hipertrofia significativa, esto explica la disociación entre la significativa disminución del S.L.G. y la leve disminución de la FEVI como en el caso presentado.

En cuanto a la aplicación clínica del strain, este tiene un rol cada vez más importante en la evaluación de los pacientes oncológicos, pero también es útil en otros escenarios, como en el estudio de pacientes que presentan engrosamiento o hipertrofia del ventrículo izquierdo sin causa evidente. Adicionalmente, la representación del strain longitudinal mediante el mapa polar permite reconocer patrones que han sido asociados a distintas patologías $^{2}$. El caso presentado evidencia un gradiente de strain longitudinal basal apical, siendo menor en los segmentos basales y medios y mayor en los segmentos apicales. Este fenómeno denominado preservación relativa del strain apical (PRSA), fue estudiado por Dermot Phelan y colaboradores ${ }^{3}$, estableciendo un adecuado rendimiento para diferenciar Amiloidosis cardíaca (independiente del subtipo) de otros tipos de hipertrofia del ventrículo izquierdo (miocardiopatía hipertrófica y estenosis aórtica) y su cálculo se resumen en la Figura 2. Se debe mencionar que este particular fenómeno de la preservación del strain apical en Amiloidosis cardíaca, también ha sido reproducido por strain mediante ecocardiografía 3D y por resonancia magnética de corazón ${ }^{4}$. Existen varias hipótesis sobre su origen, entre ellas: el menor depósito de Amiloide con menor engrosamiento y afectación de la punta del corazón y la presencia de mayor estrés hemodinámico en los segmentos basales del ventrículo izquierdo. Finalmente, señalar que la PRSA constituye una ayuda dentro de los elementos ecocardiográficos que apoyan el diagnóstico de Amiloidosis cardíaca y no constituye un criterio diagnóstico por sí solo, ya que otras patologías tales como nefropatías con hipertensión arterial no controlada de larga evolución pueden presentar PRSA, recomendando siempre la interpretación de este hallazgo de forma integrada al contexto clínico del paciente y nunca de forma aislada.

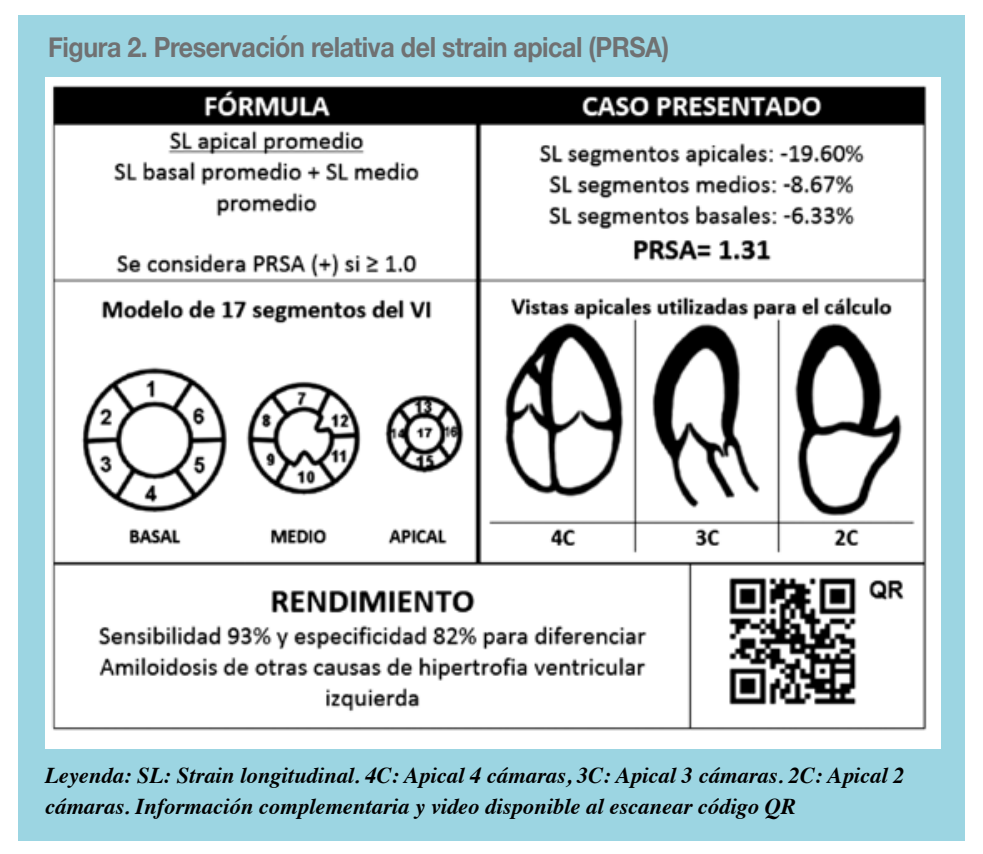


1. PINTO M. Strain: Una ventana a la mecánica ventricular. Rev Chil Cardiol. 2011;30:155-9.

2. LIU D, HU K, NORDBECK P, ERTL G, STÖRK S, WEIDEMANN F. Longitudinal strain bull's eye plot patterns in patients with cardiomyopathy and concentric left ventricular hypertrophy. Eur J Med Res. BioMed Central; 2016;21:21.

3. PHELAN D, COLLIER P, THAVENDIRANATHAN P, POPOVIĆ ZB, HANNA M, PLANA JC, et al. Relative apical sparing of longitudinal strain using two-dimensional speckle-tracking echocardiography is both sensitive and specific for the diagnosis of cardiac amyloidosis. Heart. 2012;98:1442-1448.

4. WILLIAMS LK, FORERO JF, POPOVIC ZB, PHELAN D, DELGADO D, RAKOWSKI H, et al. Patterns of CMR measured longitudinal strain and its association with late gadolinium enhancement in patients with cardiac amyloidosis and its mimics. J Cardiovasc Magn Reson. Journal of Cardiovascular Magnetic Resonance; 2017;19:61. 